PROCEEDINGS OF THE

AMERICAN MATHEMATICAL SOCIETY

Volume 124, Number 5, May 1996

\title{
BASIC DIFFERENTIAL FORMS FOR ACTIONS OF LIE GROUPS
}

\author{
PETER W. MICHOR
}

(Communicated by Roe W. Goodman)

\begin{abstract}
A section of a Riemannian $G$-manifold $M$ is a closed submanifold $\Sigma$ which meets each orbit orthogonally. It is shown that the algebra of $G$ invariant differential forms on $M$ which are horizontal in the sense that they kill every vector which is tangent to some orbit, is isomorphic to the algebra of those differential forms on $\Sigma$ which are invariant with respect to the generalized Weyl group of $\Sigma$, under some condition.
\end{abstract}

\section{INTRODUCTION}

A section of a Riemannian $G$-manifold $M$ is a closed submanifold $\Sigma$ which meets each orbit orthogonally. This notion was introduced by Szenthe [26], [27], and in a slightly different form by Palais and Terng in [19], [20]. The case of linear representations was considered by Bott and Samelson [4] and Conlon [9], and then by Dadok [10] who called representations admitting sections polar representations and completely classified all polar representations of connected compact Lie groups. Conlon [8] considered Riemannian manifolds admitting flat sections. We follow here the notion of Palais and Terng.

If $M$ is a Riemannian $G$-manifold which admits a section $\Sigma$, then the trace on $\Sigma$ of the $G$-action is a discrete group action by the generalized Weyl group $W(\Sigma)=N_{G}(\Sigma) / Z_{G}(\Sigma)$. Palais and Terng [19] showed that then the algebras of invariant smooth functions coincide, $C^{\infty}(M, \mathbb{R})^{G} \cong C^{\infty}(\Sigma, \mathbb{R})^{W(\Sigma)}$.

In this paper we will extend this result to the algebras of differential forms. Our aim is to show that pullback along the embedding $\Sigma \rightarrow M$ induces an isomorphism $\Omega_{\mathrm{hor}}^{p}(M)^{G} \cong \Omega^{p}(\Sigma)^{W(\Sigma)}$ for each $p$, where a differential form $\omega$ on $M$ is called horizontal if it kills each vector tangent to some orbit. For each point $x$ in $M$, the slice representation of the isotropy group $G_{x}$ on the normal space $T_{x}(G \cdot x)^{\perp}$ to the tangent space to the orbit through $x$ is a polar representation. The first step is to show that the result holds for polar representations. This is done in Theorem 3.7 for polar representations whose generalized Weyl group is really a Coxeter group, i.e., is generated by reflections. Every polar representation of a connected compact Lie group has this property. The method used there is inspired by Solomon [25]. Then the general result is proved under the assumption that each slice representation

Received by the editors April 6, 1994 and, in revised form November 9, 1994.

1991 Mathematics Subject Classification. Primary 57S15, 20 F55.

Key words and phrases. Orbits, sections, basic differential forms.

Supported by Project P 10037-PHY of 'Fonds zur Förderung der wissenschaftlichen Forschung'. 
has a Coxeter group as a generalized Weyl group. The last section gives some perspective to the result.

I want to thank D. Alekseevsky for introducing me to the beautiful results of Palais and Terng. I also thank A. Onishchik and D. Alekseevsky for many discussions about this and related topics, and the editor and the referees for much care and some hints.

\section{BASIC DIFFERENTIAL FORMS}

2.1. Basic differential forms. Let $G$ be a Lie group with Lie algebra $\mathfrak{g}$ and multiplication $\mu: G \times G \rightarrow G$, and for $g \in G$ let $\mu_{g}, \mu^{g}: G \rightarrow G$ denote the left and right translations.

Let $\ell: G \times M \rightarrow M$ be a left action of the Lie group $G$ on a smooth manifold $M$. We consider the partial mappings $\ell_{g}: M \rightarrow M$ for $g \in G$ and $\ell^{x}: G \rightarrow M$ for $x \in M$ and the fundamental vector field mapping $\zeta: \mathfrak{g} \rightarrow \mathfrak{X}(M)$ given by $\zeta_{X}(x)=T_{e}\left(\ell^{x}\right) X$. Since $\ell$ is a left action, the negative $-\zeta$ is a Lie algebra homomorphism.

A differential form $\varphi \in \Omega^{p}(M)$ is called $G$-invariant if $\left(\ell_{g}\right)^{*} \varphi=\varphi$ for all $g \in G$ and horizontal if $\varphi$ kills each vector tangent to a $G$-orbit: $i_{\zeta_{X}} \varphi=0$ for all $X \in \mathfrak{g}$. We denote by $\Omega_{\text {hor }}^{p}(M)^{G}$ the space of all horizontal $G$-invariant $p$-forms on $M$. They are also called basic forms.

2.2. Lemma. Under the exterior differential $\Omega_{\mathrm{hor}}(M)^{G}$ is a subcomplex of $\Omega(M)$.

Proof. If $\varphi \in \Omega_{\mathrm{hor}}(M)^{G}$, then the exterior derivative $d \varphi$ is clearly $G$-invariant. For $X \in \mathfrak{g}$ we have

$$
i_{\zeta_{X}} d \varphi=i_{\zeta_{X}} d \varphi+d i_{\zeta_{X}} \varphi=\mathcal{L}_{\zeta_{X}} \varphi=0,
$$

so $d \varphi$ is also horizontal.

2.3. Sections. Let $M$ be a connected complete Riemannian manifold, and let $G$ be a Lie group which acts isometrically on $M$ from the left. A connected closed smooth submanifold $\Sigma$ of $M$ is called a section for the $G$-action, if it meets all $G$-orbits orthogonally.

Equivalently we require that $G \cdot \Sigma=M$ and that for each $x \in \Sigma$ and $X \in \mathfrak{g}$ the fundamental vector field $\zeta_{X}(x)$ is orthogonal to $T_{x} \Sigma$.

We only remark here that each section is a totally geodesic submanifold and is given by $\exp \left(T_{x}(x \cdot G)^{\perp}\right)$ if $x$ lies in a principal orbit.

If we put $N_{G}(\Sigma):=\{g \in G: g . \Sigma=\Sigma\}$ and $Z_{G}(\Sigma):=\{g \in G: g . s=s$ for all $s \in$ $\Sigma\}$, then the quotient $W(\Sigma):=N_{G}(\Sigma) / Z_{G}(\Sigma)$ turns out to be a discrete group acting properly on $\Sigma$. It is called the generalized Weyl group of the section $\Sigma$.

See [19] or [20] for more information on sections and their generalized Weyl groups.

2.4. Main Theorem. Let $M \times G \rightarrow M$ be a proper isometric right action of a Lie group $G$ on a smooth Riemannian manifold $M$, which admits a section $\Sigma$. Let us assume that

(1) For each $x \in \Sigma$ the slice representation $G_{x} \rightarrow O\left(T_{x}(G . x)^{\perp}\right)$ has a generalized Weyl group which is a reflection group (see section 3).

Then the restriction of differential forms induces an isomorphism

$$
\Omega_{\mathrm{hor}}^{p}(M)^{G} \stackrel{\cong}{\longrightarrow} \Omega^{p}(\Sigma)^{W(\Sigma)}
$$


between the space of horizontal $G$-invariant differential forms on $M$ and the space of all differential forms on $\Sigma$ which are invariant under the action of the generalized Weyl group $W(\Sigma)$ of the section $\Sigma$.

The proof of this theorem will take up the rest of this paper. According to Dadok [10], remark after Proposition 6, for any polar representation of a connected compact Lie group the generalized Weyl group $W(\Sigma)$ is a reflection group, so condition (1) holds if we assume that:

(2) Each isotropy group $G_{x}$ is connected.

Proof of injectivity. Let $i: \Sigma \rightarrow M$ be the embedding of the section. We claim that $i^{*}: \Omega_{\mathrm{hor}}^{p}(M)^{G} \rightarrow \Omega^{p}(\Sigma)^{W(\Sigma)}$ is injective. Let $\omega \in \Omega_{\mathrm{hor}}^{p}(M)^{G}$ with $i^{*} \omega=0$. For $x \in \Sigma$ we have $i_{X} \omega_{x}=0$ for $X \in T_{x} \Sigma$ since $i^{*} \omega=0$, and also for $X \in T_{x}(G . x)$ since $\omega$ is horizontal. Let $x \in \Sigma \cap M_{\text {reg }}$ be a regular point; then $T_{x} \Sigma=\left(T_{x}(G . x)\right)^{\perp}$ and so $\omega_{x}=0$. This holds along the whole orbit through $x$ since $\omega$ is $G$-invariant. Thus $\omega \mid M_{\text {reg }}=0$, and since $M_{\text {reg }}$ is dense in $M, \omega=0$.

So it remains to show that $i^{*}$ is surjective. This will be done in 4.2 below.

\section{REPRESENTATIONS}

3.1. Invariant functions. Let $G$ be a reductive Lie group and let $\rho: G \rightarrow G L(V)$ be a representation in a finite dimensional real vector space $V$.

According to a classical theorem of Hilbert (as extended by Nagata [15], [16]), the algebra of $G$-invariant polynomials $\mathbb{R}[V]^{G}$ on $V$ is finitely generated (in fact finitely presented), so there are $G$-invariant homogeneous polynomials $f_{1}, \ldots, f_{m}$ on $V$ such that each invariant polynomial $h \in \mathbb{R}[V]^{G}$ is of the form $h=q\left(f_{1}, \ldots, f_{m}\right)$ for a polynomial $q \in \mathbb{R}\left[\mathbb{R}^{m}\right]$. Let $f=\left(f_{1}, \ldots, f_{m}\right): V \rightarrow \mathbb{R}^{m}$; then this means that the pullback homomorphism $f^{*}: \mathbb{R}\left[\mathbb{R}^{m}\right] \rightarrow \mathbb{R}[V]^{G}$ is surjective.

D. Luna proved in [14] that the pullback homomorphism $f^{*}: C^{\infty}\left(\mathbb{R}^{m}, \mathbb{R}\right) \rightarrow$ $C^{\infty}(V, \mathbb{R})^{G}$ is also surjective onto the space of all smooth functions on $V$ which are constant on the fibers of $f$. Note that the polynomial mapping $f$ in this case may not separate the $G$-orbits.

G. Schwarz proved already in [23] that if $G$ is a compact Lie group, then the pullback homomorphism $f^{*}: C^{\infty}\left(\mathbb{R}^{m}, \mathbb{R}\right) \rightarrow C^{\infty}(V, \mathbb{R})^{G}$ is actually surjective onto the space of $G$-invariant smooth functions. This result implies in particular that $f$ separates the $G$-orbits.

3.2. Lemma. Let $\ell \in V^{*}$ be a linear functional on a finite dimensional vector space $V$, and let $f \in C^{\infty}(V, \mathbb{R})$ be a smooth function which vanishes on the kernel of $\ell$, so that $f \mid \ell^{-1}(0)=0$. Then there is a unique smooth function $g$ such that $f=\ell . g$

Proof. Choose coordinates $x^{1}, \ldots, x^{n}$ on $V$ with $\ell=x^{1}$. Then $f\left(0, x^{2}, \ldots, x^{n}\right)=0$ and we have $f\left(x^{1}, \ldots, x^{n}\right)=\int_{0}^{1} \partial_{1} f\left(t x^{1}, x^{2}, \ldots, x^{n}\right) d t \cdot x^{1}=g\left(x^{1}, \ldots, x^{n}\right) \cdot x^{1}$.

3.3. Lemma. Let $W$ be a finite reflection group acting on a finite dimensional vector space $\Sigma$. Let $f=\left(f_{1}, \ldots, f_{n}\right): \Sigma \rightarrow \mathbb{R}^{n}$ be the polynomial map whose components $f_{1}, \ldots, f_{n}$ are a minimal set of homogeneous generators of the algebra $\mathbb{R}[\Sigma]^{W}$ of $W$-invariant polynomials on $\Sigma$. Then the pullback homomorphism $f^{*}$ : $\Omega^{p}\left(\mathbb{R}^{n}\right) \rightarrow \Omega^{p}(\Sigma)$ is surjective onto the space $\Omega^{p}(\Sigma)^{W}$ of $W$-invariant differential forms on $\Sigma$. 
For polynomial differential forms and more general reflection groups this is the main theorem of Solomon [25]. We adapt his proof to our needs.

Proof. The polynomial generators $f_{i}$ form a set of algebraically independent polynomials, $n=\operatorname{dim} \Sigma$, and their degrees $d_{1}, \ldots, d_{n}$ are uniquely determined up to order. We even have (see [12]):

$$
d_{1} \ldots d_{n}=|W|, \quad \text { the order of } W .
$$

$$
d_{1}+\cdots+d_{n}=n+N, \quad \text { where } N \text { is the number of reflections in } W .
$$

Let us consider the mapping $f=\left(f_{1}, \ldots, f_{n}\right): \Sigma \rightarrow \mathbb{R}^{n}$ and its Jacobian $J(x)=$ $\operatorname{det}(d f(x))$. Let $x^{1}, \ldots, x^{n}$ be coordinate functions in $\Sigma$. Then for each $\sigma \in W$ we have

$$
\begin{aligned}
J . d x^{1} \wedge \cdots \wedge d x^{n} & =d f_{1} \wedge \cdots \wedge d f_{n}=\sigma^{*}\left(d f_{1} \wedge \cdots \wedge d f_{n}\right) \\
& =(J \circ \sigma) \sigma^{*}\left(d x^{1} \wedge \cdots \wedge d x^{n}\right)=(J \circ \sigma) \operatorname{det}(\sigma)\left(d x^{1} \wedge \cdots \wedge d x^{n}\right), \\
J \circ \sigma & =\operatorname{det}\left(\sigma^{-1}\right) J .
\end{aligned}
$$

The generators $f_{1}, \ldots, f_{n}$ are algebraically independent over $\mathbb{R}$, thus $J \neq 0$. Since $J$ is a polynomial of degree $\left(d_{1}-1\right)+\cdots+\left(d_{n}-1\right)=N$ (see (2)), the $W$-invariant set $U=\Sigma \backslash J^{-1}(0)$ is open and dense in $\Sigma$; by the inverse function theorem $f$ is a local diffeomorphism on $U$, thus the 1-forms $d f_{1}, \ldots, d f_{n}$ are a coframe on $U$.

Now let $\left(\sigma_{\alpha}\right)_{\alpha=1, \ldots, N}$ be the set of reflections in $W$, with reflection hyperplanes $H_{\alpha}$. Let $\ell_{\alpha} \in \Sigma^{*}$ be linear functionals with $H_{\alpha}=\ell^{-1}(0)$. If $x \in H_{\alpha}$ we have $J(x)=\operatorname{det}\left(\sigma_{\alpha}\right) J\left(\sigma_{\alpha} \cdot x\right)=-J(x)$, so that $J \mid H_{\alpha}=0$ for each $\alpha$, and by Lemma 3.2 we have

$$
J=c . \ell_{1} \ldots \ell_{N}
$$

Since $J$ is a polynomial of degree $N, c$ must be a constant. Repeating the last argument for an arbitrary function $g$ and using (4), we get:

$$
\begin{aligned}
& \text { If } g \in C^{\infty}(\Sigma, \mathbb{R}) \text { satisfies } g \circ \sigma=\operatorname{det}\left(\sigma^{-1}\right) g \text { for each } \\
& \sigma \in W \text {, we have } g=J . h \text { for } h \in C^{\infty}(\Sigma, \mathbb{R})^{W} .
\end{aligned}
$$

After these preparations we turn to the assertion of the lemma. Let $\omega \in \Omega^{p}(\Sigma)^{W}$. Since the 1 -forms $d f_{j}$ form a coframe on $U$, we have

$$
\omega\left|U=\sum_{j_{1}<\cdots<j_{p}} g_{j_{1} \ldots j_{p}} d f_{j_{1}}\right| U \wedge \cdots \wedge d f_{j_{p}} \mid U
$$

for $g_{j_{1} \ldots j_{p}} \in C^{\infty}(U, \mathbb{R})$. Since $\omega$ and all $d f_{i}$ are $W$-invariant, we may replace $g_{j_{1} \ldots j_{p}}$ by their averages over $W$, or assume without loss that $g_{j_{1} \ldots j_{p}} \in C^{\infty}(U, \mathbb{R})^{W}$.

Let us choose now a form index $i_{1}<\cdots<i_{p}$ with $\left\{i_{p+1}<\cdots<i_{n}\right\}=$ $\{1, \ldots, n\} \backslash\left\{i_{1}<\cdots<i_{p}\right\}$. Then for some sign $\varepsilon= \pm 1$ we have

$$
\omega \mid U \wedge d f_{i_{p+1}} \wedge \cdots \wedge d f_{i_{n}}=\varepsilon \cdot g_{i_{1} \ldots i_{p}} . d f_{1} \wedge \cdots \wedge d f_{n}=\varepsilon \cdot g_{i_{1} \ldots i_{p}} . J . d x^{1} \wedge \cdots \wedge d x^{n},
$$

for a function $k_{i_{1} \ldots i_{p}} \in C^{\infty}(\Sigma, \mathbb{R})$. Thus

$$
k_{i_{1} \ldots i_{p}}\left|U=g_{i_{1} \ldots i_{p}} . J\right| U .
$$

Since $\omega$ and each $d f_{i}$ is $W$-invariant, from (6) we get $k_{i_{1} \ldots i_{p}} \circ \sigma=\operatorname{det}\left(\sigma^{-1}\right) k_{i_{1} \ldots i_{p}}$ for each $\sigma \in W$. But then by (5) we have $k_{i_{1} \ldots i_{p}}=\omega_{i_{1} \ldots i_{p}} . J$ for unique $\omega_{i_{1} \ldots i_{p}} \in$ $C^{\infty}(\Sigma, \mathbb{R})^{W}$, and $(7)$ then implies $\omega_{i_{1} \ldots i_{p}} \mid U=g_{i_{1} \ldots i_{p}}$, so that the lemma follows since $U$ is dense. 
3.4. Question. Let $\rho: G \rightarrow G L(V)$ be a representation of a compact Lie group in a finite dimensional vector space $V$. Let $f=\left(f_{1}, \ldots, f_{m}\right): V \rightarrow \mathbb{R}^{m}$ be the polynomial mapping whose components $f_{i}$ are a minimal set of homogeneous generators for the algebra $\mathbb{R}[V]^{G}$ of invariant polynomials.

We consider the pullback homomorphism $f^{*}: \Omega^{p}\left(\mathbb{R}^{m}\right) \rightarrow \Omega^{p}(V)$. Is it surjective onto the space $\Omega_{\mathrm{hor}}^{p}(V)^{G}$ of $G$-invariant horizontal smooth p-forms on $V$ ?

The proof of Theorem 3.7 below will show that the answer is yes for polar representations of compact Lie groups if the corresponding generalized Weyl group is a reflection group.

In general the answer is no. A counterexample is the following: Let the cyclic group $\mathbb{Z}_{n}=\mathbb{Z} / n \mathbb{Z}$ of order $n$, viewed as the group of $n$-th roots of unity, act on $\mathbb{C}=\mathbb{R}^{2}$ by complex multiplication. A generating system of polynomials consists of $f_{1}=|z|^{2}, f_{2}=\operatorname{Re}\left(z^{n}\right), f_{3}=\operatorname{Im}\left(z^{n}\right)$. But then each $d f_{i}$ vanishes at 0 and there is no chance to have the horizontal invariant volume form $d x \wedge d y$ in $f^{*} \Omega\left(\mathbb{R}^{3}\right)$.

3.5. Polar representations. Let $G$ be a compact Lie group and let $\rho: G \rightarrow$ $G L(V)$ be an orthogonal representation in a finite dimensional real vector space $V$ which admits a section $\Sigma$. Then the section turns out to be a linear subspace and the representation is called a polar representation, following Dadok [10], who gave a complete classification of all polar representations of connected Lie groups. They were called variationally complete representations by Conlon [9] before.

3.6. Theorem (Terng [28], Theorem D or [19], 4.12). Let $\rho: G \rightarrow G L(V)$ be a polar representation of a compact Lie group $G$, with section $\Sigma$ and generalized Weyl group $W=W(\Sigma)$. Then the algebra $\mathbb{R}[V]^{G}$ of $G$-invariant polynomials on $V$ is isomorphic to the algebra $\mathbb{R}[\Sigma]^{W}$ of $W$-invariant polynomials on the section $\Sigma$, via the restriction mapping $f \mapsto f \mid \Sigma$.

3.7. Theorem. Let $\rho: G \rightarrow G L(V)$ be a polar representation of a compact Lie group $G$, with section $\Sigma$ and generalized Weyl group $W=W(\Sigma)$. Let us suppose that $W=W(\Sigma)$ is generated by reflections (a reflection group or Coxeter group). Then the pullback to $\Sigma$ of differential forms induces an isomorphism

$$
\Omega_{\text {hor }}^{p}(V)^{G} \stackrel{\cong}{\longrightarrow} \Omega^{p}(\Sigma)^{W(\Sigma)} .
$$

According to Dadok [10], remark after Proposition 6, for any polar representation of a connected compact Lie group the generalized Weyl group $W(\Sigma)$ is a reflection group. This theorem is true for polynomial differential forms, and also for real analytic differential forms, by essentially the same proof.

Proof. Let $i: \Sigma \rightarrow V$ be the embedding. By the first part of the proof of Theorem 2.4 the pullback mapping $i^{*}: \Omega_{\mathrm{hor}}^{p}(V)^{G} \rightarrow \Omega_{\mathrm{hor}}^{p}(\Sigma)^{W}$ is injective, and we shall show that it is also surjective. Let $f_{1}, \ldots, f_{n}$ be a minimal set of homogeneous generators of the algebra $\mathbb{R}[\Sigma]^{W}$ of $W$-invariant polynomials on $\Sigma$. Then by Lemma 3.3 each $\omega \in \Omega^{p}(\Sigma)^{W}$ is of the form

$$
\omega=\sum_{j_{1}<\cdots<j_{p}} \omega_{j_{1} \ldots j_{p}} d f_{j_{1}} \wedge \cdots \wedge d f_{j_{p}}
$$

where $\omega_{j_{1} \ldots j_{p}} \in C^{\infty}(\Sigma, \mathbb{R})^{W}$. By Theorem 3.6 the algebra $\mathbb{R}[V]^{G}$ of $G$-invariant polynomials on $V$ is isomorphic to the algebra $\mathbb{R}[\Sigma]^{W}$ of $W$-invariant polynomials on 
the section $\Sigma$, via the restriction mapping $i^{*}$. Choose polynomials $\tilde{f}_{1}, \ldots \tilde{f}_{n} \in \mathbb{R}[V]^{G}$ with $\tilde{f}_{i} \circ i=f_{i}$ for all $i$. Put $\tilde{f}=\left(\tilde{f}_{1}, \ldots, \tilde{f}_{n}\right): V \rightarrow \mathbb{R}^{n}$. Then we use the theorem of G. Schwarz (see 3.1) to find $h_{i_{1}, \ldots, i_{p}} \in C^{\infty}\left(\mathbb{R}^{n}, \mathbb{R}\right)$ with $h_{i_{1}, \ldots, i_{p}} \circ f=\omega_{i_{1}, \ldots, i_{p}}$ and consider

$$
\tilde{\omega}=\sum_{j_{1}<\cdots<j_{p}}\left(h_{j_{1} \ldots j_{p}} \circ \tilde{f}\right) d \tilde{f}_{j_{1}} \wedge \cdots \wedge d \tilde{f}_{j_{p}}
$$

which is in $\Omega_{\mathrm{hor}}^{p}(V)^{G}$ and satisfies $i^{*} \tilde{\omega}=\omega$.

Sketch of another proof avoiding 3.3 (suggested by a referee). Let $R=C^{\infty}(V)^{G}=$ $C^{\infty}(\Sigma)^{W}$ and let $\Omega_{R}^{p}$ be its module of Kähler $p$-forms (see Kunz [13] for the notion of Kähler forms). Also let $S=\mathbb{R}[V]^{G}=\mathbb{R}[\Sigma]^{W}$ (using 3.6). Then the canonical mapping $\Omega_{R}^{p} \rightarrow \Omega^{p}(\Sigma)^{W}$ is surjective. This follows for the canonical mapping from $\Omega_{S}^{p}$ into the space of forms with polynomial coefficients from the result of Solomon [25] by using 3.6 again as in the proof of 3.7 ; and it can be extended to smooth coefficients by Theorem 1.4 of Ronga [22], which says that equivariant stability and infinitesimal equivariant stability are equivalent, in a way which is similar to the argument of Proposition 6.8 of Schwarz [24]. So we see that the composition $\Omega_{R}^{p} \rightarrow \Omega^{p}(V)^{G} \rightarrow \Omega^{p}(\Sigma)^{W}$ is surjective, thus also the right hand side mapping has to be surjective.

3.8. Corollary. Let $\rho: G \rightarrow O(V,\langle, \quad\rangle)$ be an orthogonal polar representation of a compact Lie group $G$, with section $\Sigma$ and generalized Weyl group $W=W(\Sigma)$. Let us suppose that $W=W(\Sigma)$ is generated by reflections (a reflection group or Coxeter group). Let $B \subset V$ be an open ball centered at 0 .

Then the restriction of differential forms induces an isomorphism

$$
\Omega_{\mathrm{hor}}^{p}(B)^{G \stackrel{\cong}{\longrightarrow}} \Omega^{p}(\Sigma \cap B)^{W(\Sigma)} .
$$

Proof. Check the proof of 3.7 or use the following argument. Suppose that $B=$ $\{v \in V:|v|<1\}$ and consider a smooth diffeomorphism $f:[0,1) \rightarrow[0, \infty)$ with $f(t)=t$ near 0 . Then $g(v):=\frac{f(|v|)}{|v|} v$ is a $G$-equivariant diffeomorphism $B \rightarrow V$ and by 3.7 we get:

$$
\Omega_{\mathrm{hor}}^{p}(B)^{G} \stackrel{\left(g^{-1}\right)^{*}}{\longrightarrow} \Omega_{\mathrm{hor}}^{p}(V)^{G} \stackrel{\cong}{\longrightarrow} \Omega^{p}(\Sigma)^{W(\Sigma)} \stackrel{g^{*}}{\longrightarrow} \Omega^{p}(\Sigma \cap B)^{W(\Sigma)} .
$$

\section{Proof of the main theorem}

Let us assume that we are in the situation of the main theorem 2.4, for the rest of this section.

4.1. For $x \in M$ let $S_{x}$ be a (normal) slice and $G_{x}$ the isotropy group, which acts on the slice. Then G.S $S_{x}$ is open in $M$ and $G$-equivariantly diffeomorphic to the associated bundle $G \rightarrow G / G_{x}$ via

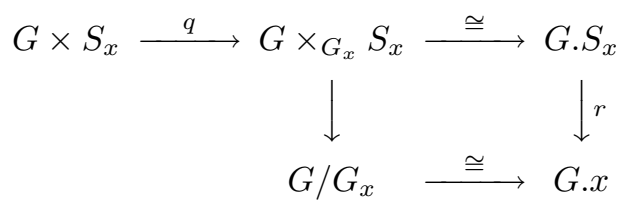


where $r$ is the projection of a tubular neighborhood. Since $q: G \times S_{x} \rightarrow G \times{ }_{G_{x}} S_{x}$ is a principal $G_{x}$-bundle with principal right action $(g, s) \cdot h=\left(g h, h^{-1} . s\right)$, we have an isomorphism

$$
q^{*}: \Omega\left(G \times_{G_{x}} S_{x}\right) \rightarrow \Omega_{G_{x}-\mathrm{hor}}\left(G \times S_{x}\right)^{G_{x}} .
$$

Since $q$ is also $G$-equivariant for the left $G$-actions, the isomorphism $q^{*}$ maps the subalgebra $\Omega_{\mathrm{hor}}^{p}\left(G . S_{x}\right)^{G} \cong \Omega_{\mathrm{hor}}^{p}\left(G \times{ }_{G_{x}} S_{x}\right)^{G}$ of $\Omega\left(G \times{ }_{G_{x}} S_{x}\right)$ to the subalgebra $\Omega_{G_{x}-\mathrm{hor}}^{p}\left(S_{x}\right)^{G_{x}}$ of $\Omega_{G_{x}-\text { hor }}\left(G \times S_{x}\right)^{G_{x}}$. So we have proved:

Lemma. In this situation there is a canonical isomorphism

$$
\Omega_{\mathrm{hor}}^{p}\left(G \cdot S_{x}\right)^{G} \stackrel{\cong}{\rightrightarrows} \Omega_{G_{x}-\mathrm{hor}}^{p}\left(S_{x}\right)^{G_{x}}
$$

which is given by pullback along the embedding $S_{x} \rightarrow G . S_{x}$.

4.2. Rest of the proof of Theorem 2.4. Now let us consider $\omega \in \Omega^{p}(\Sigma)^{W(\Sigma)}$. We want to construct a form $\tilde{\omega} \in \Omega_{\text {hor }}^{p}(M)^{G}$ with $i^{*} \tilde{\omega}=\omega$. This will finish the proof of Theorem 2.4.

Choose $x \in \Sigma$ and an open ball $B_{x}$ with center 0 in $T_{x} M$ such that the Riemannian exponential mapping $\exp _{x}: T_{x} M \rightarrow M$ is a diffeomorphism on $B_{x}$. We consider now the compact isotropy group $G_{x}$ and the slice representation $\rho_{x}: G_{x} \rightarrow O\left(V_{x}\right)$, where $V_{x}=\operatorname{Nor}_{x}(G . x)=\left(T_{x}(G . x)\right)^{\perp} \subset T_{x} M$ is the normal space to the orbit. This is a polar representation with section $T_{x} \Sigma$, and its generalized Weyl group is given by $W\left(T_{x} \Sigma\right) \cong N_{G}(\Sigma) \cap G_{x} / Z_{G}(\Sigma)=W(\Sigma)_{x}$ (see [19]); it is a Coxeter group by assumption (1) in 2.4. Then $\exp _{x}: B_{x} \cap V_{x} \rightarrow S_{x}$ is a diffeomorphism onto a slice and $\exp _{x}: B_{x} \cap T_{x} \Sigma \rightarrow \Sigma_{x} \subset \Sigma$ is a diffeomorphism onto an open neighborhood $\Sigma_{x}$ of $x$ in the section $\Sigma$.

Let us now consider the pullback $\left(\exp \mid B_{x} \cap T_{x} \Sigma\right)^{*} \omega \in \Omega^{p}\left(B_{x} \cap T_{x} \Sigma\right)^{W\left(T_{x} \Sigma\right)}$. By Corollary 3.8 there exists a unique form $\varphi^{x} \in \Omega_{G_{x}-\text { hor }}^{p}\left(B_{x} \cap V_{x}\right)^{G_{x}}$ such that $i^{*} \varphi^{x}=\left(\exp \mid B_{x} \cap T_{x} \Sigma\right)^{*} \omega$, where $i_{x}$ is the embedding. Then we have

$$
\left(\left(\exp \mid B_{x} \cap V_{x}\right)^{-1}\right) * \varphi^{x} \in \Omega_{G_{x}-\text { hor }}^{p}\left(S_{x}\right)^{G_{x}}
$$

and by Lemma 4.1 this form corresponds uniquely to a differential form $\omega^{x} \in$ $\Omega_{\text {hor }}^{p}\left(G . S_{x}\right)^{G}$ which satisfies $\left(i \mid \Sigma_{x}\right)^{*} \omega^{x}=\omega \mid \Sigma_{x}$, since the exponential mapping commutes with the respective restriction mappings. Now the intersection $G . S_{x} \cap \Sigma$ is the disjoint union of all the open sets $w_{j}\left(\Sigma_{x}\right)$ where we pick one $w_{j}$ in each left coset of the subgroup $W(\Sigma)_{x}$ in $W(\Sigma)$. If we choose $g_{j} \in N_{G}(\Sigma)$ projecting on $w_{j}$ for all $j$, then

$$
\begin{aligned}
\left(i \mid w_{j}\left(\Sigma_{x}\right)\right)^{*} \omega^{x} & =\left(\ell_{g_{j}} \circ i \mid \Sigma_{x} \circ w_{j}^{-1}\right)^{*} \omega^{x}=\left(w_{j}^{-1}\right)^{*}\left(i \mid \Sigma_{x}\right)^{*} \ell_{g_{j}}^{*} \omega^{x} \\
& =\left(w_{j}^{-1}\right)^{*}\left(i \mid \Sigma_{x}\right)^{*} \omega^{x}=\left(w_{j}^{-1}\right)^{*}\left(\omega \mid \Sigma_{x}\right)=\omega \mid w_{j}\left(\Sigma_{x}\right),
\end{aligned}
$$

so that $\left(i \mid G . S_{x} \cap \Sigma\right)^{*} \omega^{x}=\omega \mid G . S_{x} \cap \Sigma$. We can do this for each point $x \in \Sigma$.

Using the method of Palais ([18], proof of 4.3.1) we may find a sequence of points $\left(x_{n}\right)_{n \in \mathbb{N}}$ in $\Sigma$ such that the $\pi\left(\Sigma_{x_{n}}\right)$ form a locally finite open cover of the orbit space $M / G \cong \Sigma / W(\Sigma)$, and a smooth partition of unity $f_{n}$ consisting of $G$-invariant functions with $\operatorname{supp}\left(f_{n}\right) \subset G . S_{x_{n}}$. Then $\tilde{\omega}:=\sum_{n} f_{n} \omega^{x_{n}} \in \Omega_{\text {hor }}^{p}(M)^{G}$ has the required property $i^{*} \tilde{\omega}=\omega$. 


\section{BASIC VERSUS EQUIVARIANT COHOMOLOGY}

5.1. Basic cohomology. For a Lie group $G$ and a smooth $G$-manifold $M$, by 2.2 we may consider the basic cohomology $H_{G \text {-basic }}^{p}(M)=H^{p}\left(\Omega_{\mathrm{hor}}^{*}(M)^{G}, d\right)$.

The best known application of basic cohomology is the case of a compact connected Lie group $G$ acting on itself by left translations; see e.g. [11] and papers cited therein: By homotopy invariance and integration we get $H(G)=H_{G \text {-basic }}(G)=$ $H\left(\Lambda\left(\mathfrak{g}^{*}\right)\right)$, and the latter space turns out as the space $\Lambda\left(\mathfrak{g}^{*}\right)^{\mathfrak{g}}$ of $\operatorname{ad}(\mathfrak{g})$-invariant forms, using the inversion. This is the theorem of Chevalley and Eilenberg. Moreover, $\Lambda\left(\mathfrak{g}^{*}\right)^{\mathfrak{g}}=\Lambda(P)$, where $P$ is the graded subspace of primitive elements, using the Weil map and transgression, whose determination in all concrete cases by Borel and Hirzebruch is a beautiful part of modern mathematics.

In more general cases the determination of basic cohomology was more difficult. A replacement for it is equivariant cohomology, which comes in two guises:

5.2. Equivariant cohomology, Borel model. For a topological group and a topological $G$-space the equivariant cohomology was defined as follows; see [3]: Let $E G \rightarrow B G$ be the classifying $G$-bundle, and consider the associated bundle $E G \times{ }_{G} M$ with standard fiber the $G$-space $M$. Then the equivariant cohomology is given by $H^{p}\left(E G \times{ }_{G} M ; \mathbb{R}\right)$.

5.3. Equivariant cohomology, Cartan model. For a Lie group $G$ and a smooth $G$-manifold $M$ we consider the space

$$
\left(S^{k} \mathfrak{g}^{*} \otimes \Omega^{p}(M)\right)^{G}
$$

of all homogeneous polynomial mappings $\alpha: \mathfrak{g} \rightarrow \Omega^{p}(M)$ of degree $k$ from the Lie algebra $\mathfrak{g}$ of $G$ to the space of $p$-forms, which are $G$-equivariant: $\alpha\left(\operatorname{Ad}\left(g^{-1}\right) X\right)=$ $\ell_{g}^{*} \alpha(X)$ for all $g \in G$. The mapping

$$
\begin{gathered}
d_{\mathfrak{g}}: A_{G}^{q}(M) \rightarrow A_{G}^{q+1}(M), \\
A_{G}^{q}(M):=\bigoplus_{2 k+p=q}\left(S^{k} \mathfrak{g}^{*} \otimes \Omega^{p}(M)\right)^{G}, \\
\left(d_{\mathfrak{g}} \alpha\right)(X):=d(\alpha(X))-i_{\zeta_{X}} \alpha(X)
\end{gathered}
$$

satisfies $d_{\mathfrak{g}} \circ d_{\mathfrak{g}}=0$ and the following result holds.

Theorem. Let $G$ be a compact connected Lie group and let $M$ be a smooth $G$ manifold. Then

$$
H^{p}\left(E G \times_{G} M ; \mathbb{R}\right)=H^{p}\left(A_{G}^{*}(M), d_{\mathfrak{g}}\right) .
$$

This result is stated in [1] together with some arguments, and it is attributed to [5], [6] in chapter 7 of [2]. I was unable to find a satisfactory published proof.

5.4. Let $M$ be a smooth $G$-manifold. Then the obvious embedding $j(\omega)=1 \otimes \omega$ gives a mapping of graded differential algebras

$$
j: \Omega_{\mathrm{hor}}^{p}(M)^{G} \rightarrow\left(S^{0} \mathfrak{g}^{*} \otimes \Omega^{p}(M)\right)^{G} \rightarrow \bigoplus_{k}\left(S^{k} \mathfrak{g}^{*} \otimes \Omega^{p-2 k}(M)\right)^{G}=A_{G}^{p}(M) .
$$


On the other hand evaluation at $0 \in \mathfrak{g}$ defines a homomorphism of graded differential algebras ev $: A_{G}^{*}(M) \rightarrow \Omega^{*}(M)^{G}$, and $\mathrm{ev}_{0} \circ j$ is the embedding $\Omega_{\mathrm{hor}}^{*}(M)^{G} \rightarrow$ $\Omega^{*}(M)^{G}$. Thus we get canonical homomorphisms in cohomology

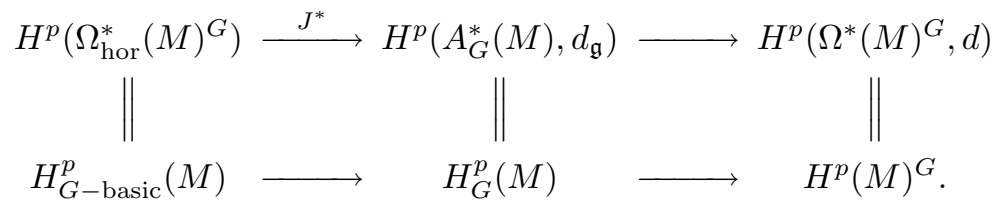

If $G$ is compact and connected we have $H^{p}(M)^{G}=H^{p}(M)$, by integration and homotopy invariance.

\section{REFERENCES}

1. Atiyah, M.; Bott, R., The moment map and equivariant cohomology, Topology 23 (1984), 1-28. MR 85e:58041

2. Berline, N.; Getzler, E.; Vergne, M., Heat kernels and differential operators, Grundlehren math. Wiss. 298, Springer-Verlag, Berlin, Heidelberg, New York, 1992. MR 94e:58130

3. Borel, A., Seminar on transformation groups, Annals of Math. Studies, Princeton Univ. Press, Princeton, 1960. MR 22:7129

4. Bott, R.; Samelson, H., Applications of the theory of Morse to symmetric spaces, Amer. J. Math. 80 (1958), 964-1029. MR 21:4430

5. Cartan, H., Notions d'algèbre differentielle; application aux groupes de Lie et aux variétés où opère un group de Lie, Colloque de Topologie, C.B.R.M., Bruxelles, 1950, pp. 15-27. MR 13:107e

6. Cartan, H., La transgression dans un group de Lie et dans un espace fibré principal, Colloque de Topologie, C.B.R.M., Bruxelles, 1950, pp. 57-71. MR 13:107f

7. Chevalley, C., Invariants of finite groups generated by reflections, Amer. J. Math. 77 (1955), 778-782. MR 17:345d

8. Conlon, L., Variational completeness and K-transversal domains, J. Differential Geom. 5 (1971), 135-147. MR 45:4320

9. Conlon, L., A class of variationally complete representations, J. Differential Geom. 7 (1972), 149-160. MR 51:14123

10. Dadok, J., Polar coordinates induced by actions of compact Lie groups, TAMS 288 (1985), 125-137. MR 86k:22019

11. Greub, Werner; Halperin, Steve; Vanstone, Ray, Connections, Curvature, and Cohomology III, Academic Press, New York and London, 1976. MR 53:4110

12. Humphreys, J. E., Reflection groups and Coxeter groups, Cambridge studies in advanced mathematics 29, Cambridge University Press, Cambridge, 1990, 1992. MR 92h:20002

13. Kunz, Ernst, Kähler Differentials, Viehweg, Braunschweig - Wiesbaden, 1986. MR 88e:14025

14. Luna, D., Fonctions différentiables invariantes sous l'operation d'un groupe réductif, Ann. Inst. Fourier, Grenoble 26 (1976), 33-49. MR 54:11377

15. Nagata, M., On the 14-th problem of Hilbert, Amer. J. Math. 81 (1959), 766-772. MR 21:4151

16. Nagata, M., Lectures on the fourteenth problem of Hilbert, Tata Inst. of Fund. Research, Bombay, 1965. MR 35:6663

17. Onishchik, A. L., On invariants and almost invariants of compact Lie transformation groups, Trudy Mosk. Math. Obshch. 35 (1976), 235-264; Trans. Moscow Math. Soc. N. 1 (1979), 237-267.

18. Palais, R., On the existence of slices for actions of non-compact Lie groups, Ann. of Math. (2) 73 (1961), 295-323. MR 23:A3802

19. Palais, R. S.; Terng, C. L., A general theory of canonical forms, Trans. AMS 300 (1987), 771-789. MR 88f:57069

20. Palais, R. S.; Terng, C. L., Critical point theory and submanifold geometry, Lecture Notes in Mathematics 1353, Springer-Verlag, Berlin, 1988. MR 90c:53143

21. Popov, V. L., Groups, generators, syzygies, and orbits in invariant theory, Translations of mathematical monographs 100, Amer. Math. Soc., Providence, 1992. MR 93g:14054 
22. Ronga, F., Stabilité locale des applications equivariantes, Differential Topology and Geometry, Dijon 1974, Lecture Notes in Math. 484, Springer-Verlag, 1975, pp. 23-35. MR 56:3866

23. Schwarz, G. W., Smooth functions invariant under the action of a compact Lie group, Topology 14 (1975), 63-68. MR 51:6870

24. Schwarz, G. W., Lifting smooth homotopies of orbit spaces, Publ. Math. IHES 51 (1980), 37-136. MR 81h:57024

25. Solomon, L., Invariants of finite reflection groups, Nagoya Math. J. 22 (1963), 57-64. MR 27:4872

26. Szenthe, J., A generalization of the Weyl group, Acta Math. Hungarica 41 (1983), 347-357. MR 85b:57044

27. Szenthe, J., Orthogonally transversal submanifolds and the generalizations of the Weyl group, Period. Math. Hungarica 15 (1984), 281-299. MR 86m:53065

28. Terng, C. L., Isoparametric submanifolds and their Coxeter groups, J. Diff. Geom. 1985 (21), 79-107. MR 87e:53095

Erwin Schrödinger International Institute of Mathematical Physics, Wien, Austria, Institut Für Mathematik, Universität Wien, Austria

E-mail address: MICHOR@ESI.AC.AT

Institut für Mathematik, Universität Wien, Strudlhofgasse 4, A-1090 Wien, Austria 